\title{
Closing the Brief Case: Retropharyngeal Abscess in a 14-Year- Old Caused by Fusobacterium necrophorum
}

\author{
Tam T. Van, ${ }^{\text {a Samia N. Naccache, }}$ 'Jennifer Dien Bard ${ }^{b, c}$ \\ aDepartment of Pathology, Harbor-UCLA Medical Center, Torrance, California, USA \\ bDepartment of Pathology and Laboratory Medicine, Children's Hospital Los Angeles, Los Angeles, \\ California, USA \\ cKeck School of Medicine, University of Southern California, Los Angeles, California, USA
}

KEYWORDS Fusobacterium, abscess, culture, pediatric, pharyngeal

\section{ANSWERS TO SELF-ASSESSMENT QUESTIONS}

1. Which of the following etiologies of bacterial pharyngitis is not detected by routine aerobic throat culture?
A. Fusobacterium necrophorum
B. Group C and G streptococci
C. Streptococcus pyogenes
D. Arcanobacterium haemolyticum

Answer: A. All of the choices have been implicated in bacterial pharyngitis; however, F. necrophorum is an obligate anaerobe whose detection requires a special request for anaerobic culture if the detection of $F$. necrophorum is not routinely performed in an institution's microbiology laboratory. Group $\mathrm{C}$ and $\mathrm{G}$ streptococci, S. pyogenes, and A. haemolyticum can be isolated from aerobic throat culture.

2. Which antimicrobial agent is most likely to be ineffective against $F$. necrophorum?
A. Penicillin
B. imipenem
C. Metronidazole
D. Erythromycin

Answer: D. Penicillin resistance among Fusobacterium spp. is relatively rare. Studies have also shown that $F$. necrophorum is susceptible to imipenem and metronidazole. Erythromycin resistance or reduced susceptibility, however, has been documented.

3. Under which conditions can F. necrophorum be isolated?
A. Blood agar incubated at $35^{\circ} \mathrm{C}$ and $5 \% \mathrm{CO}_{2}$
B. Chocolate agar incubated at $35^{\circ} \mathrm{C}$ in ambient air
C. Brucella agar incubated at $35^{\circ} \mathrm{C}$ and $5 \% \mathrm{CO}_{2}$
D. Brucella agar incubated at $35^{\circ} \mathrm{C}$ under anaerobic conditions

Answer: D. F. necrophorum is an obligate anaerobic Gram-negative bacterium that will not grow under aerobic conditions. Under the appropriate anaerobic conditions, F. necrophorum has been shown to be detectable after $24 \mathrm{~h}$ of

Citation Van TT, Naccache SN, Dien Bard J. 2018. Closing the Brief Case: Retropharyngeal abscess in a 14-year-old caused by Fusobacterium necrophorum. J Clin Microbiol 56:e00488-18. https://doi.org/10.1128/JCM .00488-18.

Editor Carey-Ann D. Burnham, Washington University School of Medicine

Copyright $\odot 2018$ American Society for Microbiology. All Rights Reserved.

Address correspondence to Jennifer Dien Bard, jdienbard@chla.usc.edu.

T.T.V. and S.N.N. contributed equally to the manuscript.

See https://doi.org/10.1128/JCM.00487-18 in this issue for case presentation and discussion. 
incubation. In many cases, growth is not visible until at least $48 \mathrm{~h}$ of incubation.

\section{TAKE-HOME POINTS}

- Microbiology laboratories should routinely attempt to resolve discrepancies between Gram stain and culture results. A high suspicion of an anaerobic pathogen warrants consultation with a laboratory expert and communication with the clinician immediately to maximize the recovery of anaerobes.

- Anaerobic infections are underdiagnosed. Continuing education and communication with clinicians are warranted to increase awareness and the likelihood that the appropriate specimen is submitted to the microbiology laboratory for anaerobic culture.

- F. necrophorum infection should be considered in adolescents and young adults presenting with pharyngitis who test negative for beta-hemolytic streptococci.

- There are no standard treatment guidelines for F. necrophorum. 